\title{
Chemical constituents, antibacterial and antioxidant properties of the essential oil flower of Tagetes minuta grown in Cala community Eastern Cape, South Africa
}

\author{
Aboi Igwaran ${ }^{1,2^{*}}$, Benson Chucks Iweriebor ${ }^{1,2}$, Sunday Ofuzim Okoh², Uchechukwu Uchechukwu Nwodo ${ }^{1,2}$,
} Larry Chikwelu $\mathrm{Obi}^{3}$ and Anthony Ifeanyi Okoh ${ }^{1,2}$

\begin{abstract}
Background: Tagetes minuta has a long record of human use for the treatment of stomach and intestinal diseases. Most drugs used for diseases treatment are less efficacious with side effects and this brought the search for new treatment regimens mainly from medicinal plants.

Method: The essential oil (EO) was extracted by Clevenger's-type apparatus and its chemical composition, antioxidant and antibacterial properties were determined by GC-MS, spectrophotometric and broth dilution methods respectively. S. uberis, E. cloacae, S. aureus, M. smegmatis, L. ivanovii, Vibrio spp. and E. coli bacteria strains were used as test bacteria.
\end{abstract}

Results: GC-MS analysis revealed 98 compounds in the EO flower of T. minuta and $\beta$-Ocimene (14. 40\%) was the major chemical constituents. The EO exhibited highest inhibitory effect against DPPH radical, followed by its effect on ABTS, while LP radical showed the least sensitivity with $I_{50}$ values of $2.45 \mathrm{mg} / \mathrm{mL}, 2.76 \mathrm{mg} / \mathrm{mL}$ and $3.23 \mathrm{mg} / \mathrm{mL}$ respectively. The EO showed antibacterial activities against all test organisms with MIC value for S. aureus, M. smegatis and S. uberis at $0.125 \mathrm{mg} / \mathrm{mL}$ and for L. ivanovii, Vibrio spp., E. cloacae and E. coli at $0.06 \mathrm{mg} / \mathrm{mL}$. The EO showed MBC against $E$. cloacae and E. coli at $0.06 \mathrm{mg} / \mathrm{mL}$ at $0.5 \mathrm{mg} / \mathrm{mL}$ for S. uberis and $0.125 \mathrm{mg} / \mathrm{mL}$ for Vibrio spp.

Conclusion: Findings from this study suggest that the EO of T. minuta flower may be a useful candidate in the search for lead constituents for the synthesis of new potent antibacterial and antioxidant agent.

Keywords: Antibacterial, Antioxidant, Tegates minuta, Vitamin C

\section{Background}

Free radicals instigate oxidative damage and when this free radical in the body system is above the capacity of antioxidant, it results in oxidative stress which is implicated in many human diseases [1]. Antioxidant is substance that helps to prevent oxidative damage when found in small amounts and is equivalent to an oxidizable substrate. Antioxidants aid in averting of

\footnotetext{
* Correspondence: aboi.igwaran@yahoo.com

${ }^{1}$ SAMRC Microbial Water Quality Monitoring Centre, University of Fort Hare, Alice 5700, South Africa

${ }^{2}$ Applied and Environmental Microbiology Research Group (AEMREG), Department of Biochemistry and Microbiology, University of Fort Hare, Private Bag X1314, Alice, Eastern Cape Province 5700, South Africa

Full list of author information is available at the end of the article
}

diseases by scavenging radicals including lipid peroxyl (LP •), superoxide $\left(\mathrm{O}_{2} \bullet\right.$ ), nitric oxide ( $\mathrm{NO} \bullet$ ) and hydroxyl ( $\mathrm{HO} \bullet$ ) formed during metabolic activities [2]. The need for natural antioxidants is becoming imperative due to numerous health risk associated with synthetic antioxidants [3]. Several in vitro assays such as DPPH, ABTS, lipid peroxyl and ferric reducing ability of plasma (FRAP) radicals have been used in evaluating antioxidants capacity of plants extracts which are designed based on quenching stable free radicals [4]. Plants used traditionally are known to produce a wide range of compounds with therapeutic properties such as antioxidant, antibacterial, gastroprotective effects amongst others [5]. 
Study has shown that the continuous use of synthetic antibiotics for a long period is one of the causes of bacterial resistant [6]. The treatment failures associated with multidrug-resistant bacterial strains including methicillin-resistant Staphylococcus aureus (MRSA), vancomycin-resistant Staphylococcus aureus (VRSA), vancomycin-resistant Enterococcus faecalis, Vibrio Spp. and Escherichia coli have become a worldwide concern [7], which has heightened the search for alternative therapeutic agents [8]. The use of herbal medicine for the treatment of diseases has been stated by World Health Organization (WHO) and presently a lot of persons use herbal medicine for treatment of diverse disease $[9,10]$. Plant extracts provide boundless opportunities for such option as well as in discovery of new drugs because of availability of potent chemical components present in their extracts $[11,12]$. The active compound found in medicinal plants that exhibit therapeutic activities against pathogens have little or no side effects on the host cells [13]. Phytochemical compounds present in the essential oil (EO) of plants are diverse, complicated and are known to contain active antibacterial property [14] and antioxidant properties [15]. Essential oils from medicinal plants contain naturally occurring antimicrobial compounds which have been shown to be effective in limiting the growth and survival of many pathogens [16]. These essential oils from plant such as Eucalyptus, Pogostemon cablin and tea tree have been reported to exhibit antimicrobial potential [17], and this make EO appropriate option to synthetic antibiotics [18].

Tagetes minuta is commonly called wild marigold also known as Mexican marigold of the family Asteraceae [19], and it belongs to one of the 56 species of Tagetes [20]. T. minuta is found in many countries such as Argentina and South America [21], including South Africa. T. minuta is commonly called nnkayo by the Xkosa people, a tribe in the Easter Cape Province, South Africa. T. minuta has been reported to have a long record of human use for insect repellent, treatment of stomach and intestinal diseases [22]. There has been heightened interest of late in plant-based natural products that have the ability to reduce free radicals formation and treating infections caused by pathogenic microorganisms. There is however scarce information on antibacterial and antioxidant properties as well as the chemical constituents of the essential oil flower of T. minuta grown in Cala community, Eastern Cape Province, South Africa that is claimed to be potent against many infections. This study therefore aimed to evaluate antibacterial, antioxidant properties and the chemical constituents of the essential oil of T. minuta flower grown in Cala community South Africa.

\section{Methods}

\section{Plant material}

Fresh flowers of T. minuta were collected from Cala community located in Sakhisizwe Local Municipality Eastern Cape Province, South Africa with geographical coordinates of $31^{\circ} 33^{\prime} 0^{\prime \prime}$ South, and $27^{\circ} 36^{\prime} 0^{\prime \prime}$ East [23]. Taxonomical identification of the plant was confirmed at.

Selmer Schonland Herbarium, Albany Museum Grahams town with Voucher No. BM01-040/2007 and the voucher specimen was deposited. Prior to essential oil extraction, plant material was rinsed with distilled water and shade dried on foil paper in the laboratory at ambient temperature for 6 days and thereafter, $T$. minuta flower was pulverized in a blending machine (Polymix PX-MFC90 D, Lasec/SA).

\section{Extraction of essential oil}

The essential oil obtained was extracted from the powdered flower (376.86 g) for $3 \mathrm{~h}$ with a modified hydrodistillation Clevenger's-type apparatus as described by Omoruyi et al., [24]. The hydro-distillation experiment was carried out thrice to obtain enough oil for bioactivity assays and the extracted essential oil was dried over anhydrous sodium sulphate, dispensed into tinted vials and stored at $4{ }^{\circ} \mathrm{C}$. The yield of the essential oil was determined in $w / w \%$ (per gram) of the extracted plant sample.

\section{Gas chromatography mass spectrometry (GC-MS)}

GC-MS analyses of the essential oil was performed on Agilent 5977A MSD and 7890B GC system, Chemetrix (pty) Ltd.; Agilent Technologies, DE (Germany) with a Zebron-5MS column (ZB-5MS $30 \mathrm{~m} \times 0.25 \mathrm{~mm} \times 0.25$ um) (5\% -phenylmethylpolysiloxane). The following column and temperature conditions used were: GC grade helium at a flow rate of $2 \mathrm{~mL} / \mathrm{min}$ and splitless $1 \mathrm{~mL}$ injections was used. The injector, source and oven temperatures were set at $280{ }^{\circ} \mathrm{C}, 280{ }^{\circ} \mathrm{C}$ and $70{ }^{\circ} \mathrm{C}$, respectively. The ramp settings were set at; $15{ }^{\circ} \mathrm{C} / \mathrm{min}$ to $120{ }^{\circ} \mathrm{C}$, afterwards $10{ }^{\circ} \mathrm{C} / \mathrm{min}$ to $180{ }^{\circ} \mathrm{C}$, then $20{ }^{\circ} \mathrm{C} / \mathrm{min}$ to $270{ }^{\circ} \mathrm{C}$ and held for $3 \mathrm{~min}$.

\section{Chemical reagents}

The chemicals used were acetic acid, thiobarbituric acid (TBA), trychloroacetic acid, sodium dodecyl sulphate (SDS), 2, 2-diphenyl-1-picrylhydrazyl (DPPH), 2, 2'-azino-bis-3-ethylbenzthiazoline-6-sulphonic acid (ABTS), ethanol, methanol, ethyl acetate, n-hexane, Iron (II) sulphate $\left(\mathrm{FeSO}_{4}\right)$, sodium hydroxide $(\mathrm{NaOH})$, ascorbic acid and butanol. All chemicals reagents were of analytical quality and were bought from reliable commercial sources. 


\section{Determination of antioxidant property}

Antioxidant property of the extracted essential oil of $T$. minuta flower was evaluated in vitro by spectrophotometric method against DPPH, ABTS and lipid peroxyl radicals.

\section{DPPH radical scavenging assay}

DPPH assay was carried out following the modified method of Ajileye et al., [25]. Briefly, the solution of DPPH $(0.135 \mathrm{mM})$ was prepared in methanol and incubated in the dark for $30 \mathrm{~min}$. One hundred microliter of the essential oil or standards (positive control) were prepared in methanol of various concentration ranging from $(0.03-0.5 \mathrm{mg} / \mathrm{mL})$, and was added into all the wells of microtiter plate starting from $(\mathrm{C}-\mathrm{H})$ in triplicates except for A (A1-A12) and B (B1-B12). Thereafter, $100 \mu \mathrm{L}$ of $0.135 \mathrm{mM}$ of DPPH solution prepared in methanol was added into the wells from $\mathrm{C}-\mathrm{H}$. Absorbance was spectrophotometrically observed at $517 \mathrm{~nm}$ and the essential oil ability to lower DPPH to neutral molecule was expressed as percentage inhibition using the formula percentage inhibition $=\{(\mathrm{Abs}$ control $-\mathrm{Abs}$ sample $)\} /(\mathrm{Abs}$ control) $\times 100$.

\section{ABTS scavenging assay}

ABTS test was determined following the modified method of Kannan et al., [26]. Briefly, stock solutions was prepared by mixing two stock solutions $(1: 1 \mathrm{v} / \mathrm{v})$ ratio of potassium persulfate $(2.45 \mathrm{mM})$ and ABTS $(7.0 \mathrm{mM})$, incubated in a dark cupboard for $720 \mathrm{~min}$ at ambient temperature. One microliter of ABTS+ solution was diluted by adding methanol to obtain an absorbance of $0.708 \pm 0.002$ unites when measured at $734 \mathrm{~nm}$ with spectrophotometer. Briefly, $100 \mu \mathrm{L}$ of the essential oil or standards prepared in methanol of various concentration ranging from $0.03-0.5 \mathrm{mg} / \mathrm{mL}$ was added into all the wells of microtiter plate starting from $(\mathrm{C}-\mathrm{H})$ in triplicates except for $A$ (A1-A12) and B (B1-B12). Thereafter, $100 \mu \mathrm{L}$ of ABTS + solution was then added to all the wells starting from $\mathrm{C}-\mathrm{H}$ and the solution was incubated for $7 \mathrm{~min}$ and absorbance was taken at $734 \mathrm{~nm}$ using spectrophotometer. The percentage inhibition was calculated using the formula stated above.

\section{Lipid peroxidation by TBARS test}

The modified method of thiobarbituric acid reactive species (TBARS) assay described by Badmus et al., [27] was adapted to measure the inhibitory effect of the essential oil on lipid peroxidation using egg yolk homogenates as lipid rich source. A volume of $125 \mu \mathrm{L}$ of $10 \%$ of the egg homogenate (in distilled water) was added to various concentrations ranged from $0.03-0.5 \mathrm{mg} / \mathrm{mL}$ of the plant extract prepared in methanol. The volume was adjusted to $250 \mu \mathrm{L}$ with distilled water. Afterward, $12.5 \mu \mathrm{L}$ of $\mathrm{FeSO}_{4}$ (Iron (II) sulphate) was added to the solution and incubated at $25{ }^{\circ} \mathrm{C}$ for $30 \mathrm{~min}$. A $375 \mu \mathrm{L}$ of $10 \%$ acetic acid (pH 3.50) adjusted with $\mathrm{NaOH}$. Then, $0.80 \%$ of 2-thiobarbituric acid $(375 \mu \mathrm{L})$ mixed with sodium dodecyl sulphate $1.1 \%$ and $2 \%$ trichloroacetic acid $(12.5 \mu \mathrm{L})$ was added in the same micro centrifuge tubes, vortexed and heat at $65{ }^{\circ} \mathrm{C}$ for $60 \mathrm{~min}$. After cooling, $975 \mu \mathrm{L}$ of butanol was added, centrifuged at $3000 \mathrm{rpm}$ for $600 \mathrm{~s}$. The upper organic layer was then aspirated and the absorbance read at $532 \mathrm{~nm}$. Percentage inhibition of lipid by the EO was calculated using the formula previously stated above and all the assays were performed in triplicate.

\section{Antibacterial test}

\section{Test organisms}

The reference bacteria strains used are Staphylococcus aureus (ATCC 29213), Enterobacter cloacae (ATCC 13047), Mycobacterium smegmatis (ATCC 19420), Listeria ivanovii (ATCC 19119), Streptococcus uberis (ATCC 29213), and laboratory identified Vibrio spp. and Escherichia coli.

\section{Bacteria culture condition}

Antibacterial potential of the essential oil of $T$. minuta flower was tested against four Gram-positive bacteria reference strains which were $S$. aureus, $M$. smegmatis, $S$. uberis and $L$. ivanovii and three Gram-negative bacteria namely E. cloacae, Vibrio spp. and E. coli following CLSI [28] guideline. The bacterial suspensions were made by inoculating a fresh stock culture of the test bacteria strains into tubes containing $5 \mathrm{~mL}$ of sterile LuriaBertani broth and incubated for $24 \mathrm{~h}$ at $37{ }^{\circ} \mathrm{C}$. Active cultures grown overnight in sterile Luria- Bertani broth were inoculated into Mueller-Hinton Agar (MHA) incubated for $24 \mathrm{~h}$ at $37{ }^{\circ} \mathrm{C}$. After incubation, single colonies were transferred from MHA plates into $4 \mathrm{~mL}$ of normal saline solution determined spectrophotometrically at $580 \mathrm{~nm}$ as previously reported by Duarte et al., [29] adapted by Omoruyi et al. [24], and the dilutions matching with 0.5 Mc-Farland standard were used for the assay.

\section{Determination of antibacterial properties}

The modified method of Gullon et al., [30] was adopted for the determination of MIC and MBC of the essential

Table 1 Physio-chemical characteristics of the essential oil extracted from T. minuta flower

\begin{tabular}{ll}
\hline Item & $\begin{array}{l}\text { Physico-chemical characteristics } \\
\text { of EO of } T \text {. minuta flower }\end{array}$ \\
\hline Percentage yield & $0.33 \% \mathrm{w} / \mathrm{w} \%$ \\
Colour & Very pale yellow \\
Fragrance (odour) & Pungent odour \\
\hline
\end{tabular}


Table 2 Chemical composition of the essential oils of T. minuta flower

\begin{tabular}{|c|c|c|c|c|}
\hline $\mathrm{S} / \mathrm{N}$ & Chemical constituents & Chemical formula & $\mathrm{RT}$ (min) & $\%$ Composition \\
\hline 1 & Butanoic acid, 2-methyl-, ethyl ester & $\mathrm{C}_{7} \mathrm{H}_{14} \mathrm{O}_{2}$ & 3.26 & 0.06 \\
\hline 2 & 2-Hexenal, (E)- & $\mathrm{C}_{6} \mathrm{H}_{10} \mathrm{O}$ & 3.30 & 0.09 \\
\hline 3 & 1-Hexanol & $\mathrm{C}_{6} \mathrm{H}_{14} \mathrm{O}$ & 3.37 & 0.09 \\
\hline 4 & Heptanal & $\mathrm{C}_{7} \mathrm{H}_{14} \mathrm{O}$ & 3.643 & 0.05 \\
\hline 5 & 2-Cyclopenten-1-one, 3,4-dimethyl- & $\mathrm{C}_{7} \mathrm{H}_{10} \mathrm{O}$ & 3.752 & 0.07 \\
\hline 6 & 1H-Imidazole, 2-ethyl-4-methyl- & $\mathrm{C}_{6} \mathrm{H}_{10} \mathrm{~N}_{2}$ & 3.832 & 1.29 \\
\hline 7 & Phenol, 3,4-dimethyl- & $\mathrm{C}_{7} \mathrm{H}_{10} \mathrm{O}$ & 3.923 & 0.07 \\
\hline 8 & (1S)-2,6,6-Trimethylbicyclo [3.1.1] hept-2-ene & $\mathrm{C}_{10} \mathrm{H}_{16}$ & 3.979 & 0.17 \\
\hline 9 & Nona-3,5-dien-2-one & $\mathrm{C}_{9} \mathrm{H}_{14} \mathrm{O}$ & 4.022 & 0.07 \\
\hline 10 & Camphene & $\mathrm{C}_{10} \mathrm{H}_{16}$ & 4.16 & 0.13 \\
\hline 11 & 1-Octene & $\mathrm{C}_{8} \mathrm{H}_{16}$ & 4.158 & 0.08 \\
\hline 12 & Bicyclo [3.1.0] hexane, 4-methylene- 1-(1-methylethyl)- & $\mathrm{C}_{10} \mathrm{H}_{16}$ & 4.286 & 0.39 \\
\hline 13 & Furan, 2-pentyl- & $\mathrm{C}_{9} \mathrm{H}_{14} \mathrm{O}$ & 4.374 & 0.41 \\
\hline 14 & Octanal & $\mathrm{C}_{8} \mathrm{H}_{16} \mathrm{O}$ & 4.457 & 0.36 \\
\hline 15 & a-Phellandrene & $\mathrm{C}_{10} \mathrm{H}_{16}$ & 4.537 & 0.23 \\
\hline 16 & 2,4-Heptanedione, 6-methyl- & $\mathrm{C}_{8} \mathrm{H}_{14} \mathrm{O}_{2}$ & 4.648 & 0.14 \\
\hline 17 & $\beta$-Ocimene & $\mathrm{C}_{10} \mathrm{H}_{16}$ & 4.751 & 14.40 \\
\hline 18 & 1,3,7-Octatriene, 3,7-dimethyl- & $\mathrm{C}_{10} \mathrm{H}_{16}$ & 4.843 & 0.57 \\
\hline 19 & Valeric acid, 3-tridecyl ester & $\mathrm{C}_{18} \mathrm{H}_{36} \mathrm{O}_{2}$ & 4.903 & 9.24 \\
\hline 20 & ү-Terpinene & $\mathrm{C}_{10} \mathrm{H}_{16}$ & 4.982 & 0.41 \\
\hline 21 & 3,4-Dimethylbenzyl alcohol & $\mathrm{C}_{9} \mathrm{H}_{12} \mathrm{O}$ & 5.039 & 0.07 \\
\hline 22 & Furan, 2,3-dihydro-3-methyl- & $\mathrm{C}_{5} \mathrm{H}_{8} \mathrm{O}$ & 5.066 & 0.15 \\
\hline 23 & p-Cymen-7-ol & $\mathrm{C}_{10} \mathrm{H}_{14} \mathrm{O}$ & 5.224 & 1.03 \\
\hline 24 & 6-Methyl-3,5-heptadiene-2-one & $\mathrm{C}_{8} \mathrm{H}_{12} \mathrm{O}$ & 5.299 & 0.26 \\
\hline 25 & Furazan, 3-(dimethylaminomethylenamino)-4-(1,2,4-triazol-3-yl)- & $\mathrm{C}_{7} \mathrm{H}_{9} \mathrm{~N}_{7} \mathrm{O}$ & 5.370 & 0.24 \\
\hline 26 & 2,6-Dimethyl-1,3,5,7-octatetraene, E,E- & $\mathrm{C}_{10} \mathrm{H}_{14}$ & 5.435 & 0.07 \\
\hline 27 & 2,4,6-Octatriene, 2,6-dimethyl-,E,Z)- & $\mathrm{C}_{10} \mathrm{H}_{16}$ & 5.484 & 1.88 \\
\hline 28 & 5,7-Octadien-4-one, 2,6-dimethyl-, (Z)- & $\mathrm{C}_{10} \mathrm{H}_{16} \mathrm{O}$ & 5.617 & 1.50 \\
\hline 29 & 5,7-Octadien-4-one, 2,6-dimethyl-, (E)- & $\mathrm{C}_{10} \mathrm{H}_{16} \mathrm{O}$ & 5.698 & 7.14 \\
\hline 30 & Sorbic acid vinyl ester & $\mathrm{C}_{8} \mathrm{H}_{10} \mathrm{O}_{2}$ & 5.763 & 0.34 \\
\hline 31 & Butanoic acid, 3-hexenyl ester, (E)- & $\mathrm{C}_{8} \mathrm{H}_{18} \mathrm{O}_{2}$ & 5.923 & 2.21 \\
\hline 32 & Bicyclo [2.2.1] heptane, 7,7-dimethy 1-2-methylene- & $\mathrm{C}_{10} \mathrm{H}_{16}$ & 6.028 & 0.62 \\
\hline 33 & Decanal & $\mathrm{C}_{10} \mathrm{H}_{20} \mathrm{O}$ & 6.066 & 0.64 \\
\hline 34 & Cyclopentanone, 2-cyclopentylidene & $\mathrm{C}_{10} \mathrm{H}_{14} \mathrm{O}$ & 6.121 & 0.43 \\
\hline 35 & 2-Cyclohexen-1-ol, 2-methyl-5-(1-m ethylethenyl)-, cis & $\mathrm{C}_{10} \mathrm{H}_{16} \mathrm{O}$ & 6.246 & 0.22 \\
\hline 36 & Phenol, m-tert-butyl- & $\mathrm{C}_{10} \mathrm{H}_{14} \mathrm{O}$ & 6.318 & 9.41 \\
\hline 37 & Naphthalene, 1,2,3,4,4a,5,6,7-octa hydro-4a-methyl- & $\mathrm{C}_{11} \mathrm{H}_{18}$ & 6.380 & 5.58 \\
\hline 38 & Phenol, 2,3,5,6-tetramethyl- & $\mathrm{C}_{10} \mathrm{H}_{14} \mathrm{O}$ & 6.494 & 0.60 \\
\hline 39 & 4-Methyl-1,3-heptadiene & $\mathrm{C}_{10} \mathrm{H}_{14} \mathrm{O}$ & 6.573 & 0.26 \\
\hline 40 & Orcinol & $\mathrm{C}_{7} \mathrm{H}_{8} \mathrm{O}_{2}$ & 6.626 & 0.45 \\
\hline 41 & 1H-Pyrazole, 4,5-dihydro-5,5-dimethyl-4-isopropylidene- & $\mathrm{C}_{8} \mathrm{H}_{14} \mathrm{~N}_{2}$ & 6.659 & 0.76 \\
\hline 42 & Bornyl acetate & $\mathrm{C}_{12} \mathrm{H}_{20} \mathrm{O}$ & 6.750 & 0.29 \\
\hline 43 & Propanoic acid, 2-methyl-, octyl ester & $\mathrm{C}_{12} \mathrm{H}_{14} \mathrm{O}_{2}$ & 7.032 & 0.06 \\
\hline 44 & 1,5,5-Trimethyl-6-methylene-cyclohexene & $\mathrm{C}_{10} \mathrm{H}_{16}$ & 7.128 & 0.15 \\
\hline
\end{tabular}


Table 2 Chemical composition of the essential oils of T. minuta flower (Continued)

\begin{tabular}{|c|c|c|c|c|}
\hline 45 & 2,6,10,14-Hexadecatetraen-1-ol, 3, 7,11,15-tetramethyl-, acetate, (E, E,E)- & $\mathrm{C}_{20} \mathrm{H}_{34} \mathrm{O}$ & 7.311 & 0.06 \\
\hline 46 & 2-Isopropylidene-3-methylhexa-3,5-dienal & $\mathrm{C}_{10} \mathrm{H}_{14}$ & 7.428 & 0.18 \\
\hline 47 & 1-Benzothiepin, 2,3,4,5-tetrahydro & $\mathrm{C}_{9} \mathrm{H}_{10} \mathrm{O}_{2} \mathrm{~S}$ & 7.503 & 1.23 \\
\hline 48 & Nonyl 2-methylbutanoate & $\mathrm{C}_{14} \mathrm{H}_{28} \mathrm{O}_{2}$ & 7.640 & 0.25 \\
\hline 49 & Caryophyllene & $\mathrm{C}_{15} \mathrm{H}_{24}$ & 7.756 & 1.86 \\
\hline 50 & Humulene & $\mathrm{C}_{15} \mathrm{H}_{24}$ & 7.977 & 0.73 \\
\hline 51 & Naphthalene, 1,2,3,5,6,7,8,8a-octa hydro-1,8a-dimethyl-7-(1-methylethenyl)-, [1R-(1a,7ß,8aa)]- & $\mathrm{C}_{15} \mathrm{H}_{24}$ & 8.073 & 0.25 \\
\hline 52 & Aromandendrene & $\mathrm{C}_{15} \mathrm{H}_{24}$ & 8.092 & 0.32 \\
\hline 53 & 1,6-Cyclodecadiene, 1-methyl-5-methylene-8-(1-methylethyl)-, [S-(E,E)]- & $\mathrm{C}_{15} \mathrm{H}_{26}$ & 8.142 & 0.51 \\
\hline 54 & Bicyclogermacrene & $\mathrm{C}_{15} \mathrm{H}_{24}$ & 8.239 & 1.19 \\
\hline 55 & ૪ -Muurolene & $\mathrm{C}_{15} \mathrm{H}_{24}$ & 8.327 & 0.17 \\
\hline 56 & Naphthalene, 1,2,3,5,6,8a-hexahydro-4,7-dimethyl-1-(1-methylethyl)-, (1S-cis)- & $\mathrm{C}_{15} \mathrm{H}_{24}$ & 8.351 & 0.15 \\
\hline 57 & 2,2,6-Trimethyl-1-(3-methylbuta-1, 3-dienyl)-7-oxabicyclo [4.1.0] heptan-3-ol & $\mathrm{C}_{14} \mathrm{H}_{22}$ & 8.423 & 0.60 \\
\hline 58 & 1,6,10-Dodecatrien-3-ol, 3,7,11-trimethyl-, [S-(Z)]- & $\mathrm{C}_{15} \mathrm{H}_{24} \mathrm{O}$ & 8.516 & 0.17 \\
\hline 59 & Culmorin & $\mathrm{C}_{15} \mathrm{H}_{24} \mathrm{O}_{2}$ & 8.566 & 0.02 \\
\hline 60 & Tricyclo[2.2.1.0 (2,6)] heptane, 1,7-dimethyl-7-(4-methyl-3-pentenyl)-, (-)- & $\mathrm{C}_{15} \mathrm{H}_{24}$ & 8.698 & 0.19 \\
\hline 61 & (-)-Spathulenol & $\mathrm{C}_{15} \mathrm{H}_{24} \mathrm{O}$ & 8.767 & 4.56 \\
\hline 62 & Caryophyllene oxide & $\mathrm{C}_{15} \mathrm{H}_{24} \mathrm{O}$ & 8.813 & 1.01 \\
\hline 63 & 1H-Cycloprop[e]azulen-4-ol, decahydro-1,1,4,7-tetramethyl-, [1aR-(1aa, 4ß,4aß,7a, 7aß, 7ba]- & $\mathrm{C}_{15} \mathrm{H}_{26} \mathrm{O}$ & 8.865 & 0.25 \\
\hline 64 & Levomenol & $\mathrm{C}_{15} \mathrm{H}_{26} \mathrm{O}$ & 8.914 & 0.30 \\
\hline 65 & 3-Cyclohexen-1-carboxaldehyde, 3,4-dimethyl- & $\mathrm{C}_{9} \mathrm{H}_{14} \mathrm{O}$ & 8.965 & 0.22 \\
\hline 66 & Tricyclo [4.4.0.0(2,7)] dec-3-ene-3-methanol, 1-methyl-8-(1-methylethyl)- & $\mathrm{C}_{15} \mathrm{H}_{24} \mathrm{O}$ & 9.092 & 1.06 \\
\hline 67 & Longipinocarveol, trans- & $\mathrm{C}_{15} \mathrm{H}_{24} \mathrm{O}$ & 9.293 & 0.33 \\
\hline 68 & Octadecanal & $\mathrm{C}_{18} \mathrm{H}_{36} \mathrm{O}$ & 9.358 & 0.11 \\
\hline 69 & 7-Tetracyclo [6.2.1.0(3.8)0(3.9)] undecanol, 4,4,11,11- tetramethyl & $\mathrm{C} 15 \mathrm{H} 24 \mathrm{O}$ & 9.379 & 0.11 \\
\hline 70 & Phenol, 2-methyl-4-(1,1,3,3-tetramethylbutyl)- & $\mathrm{C}_{15} \mathrm{H}_{24} \mathrm{O}$ & 9.501 & 0.12 \\
\hline 71 & Carbonic acid, 4-isopropylphenyl propargyl ester & $\mathrm{C}_{17} \mathrm{H}_{14} \mathrm{O}_{4}$ & 9.551 & 0.06 \\
\hline 72 & 11-Isopropylidenetricyclo[4.3.1.1 (2,5)]undec-3-en-10-one & $\mathrm{C}_{14} \mathrm{H}_{18}$ & 9.599 & 0.08 \\
\hline 73 & Shyobunone & $\mathrm{C}_{15} \mathrm{H}_{24} \mathrm{O}$ & 9.647 & 0.03 \\
\hline 74 & 2-Acetyl-6-methoxynaphthalene & $\mathrm{C}_{13} \mathrm{H}_{12} \mathrm{O}_{2}$ & 9.828 & 0.14 \\
\hline 75 & Cholesterol & $\mathrm{C}_{27} \mathrm{H}_{46} \mathrm{O}$ & 9.879 & 0.04 \\
\hline 76 & Bicyclo[3.1.1]heptane, 2,6,6-trimethyl-,(1a,2ß,5a) & $\mathrm{C}_{10} \mathrm{H}_{18}$ & 10.020 & 0.63 \\
\hline 77 & 2-Pentadecanone, 6,10,14-trimethyl & $\mathrm{C}_{18} \mathrm{H}_{36} \mathrm{O}$ & 10.060 & 0.53 \\
\hline 78 & Caryophyllene oxide & $\mathrm{C}_{15} \mathrm{H}_{24} \mathrm{O}$ & 10.124 & 0.15 \\
\hline 79 & 1-Hexadecanol & $\mathrm{C}_{16} \mathrm{H}_{34} \mathrm{O}$ & 10.230 & 0.22 \\
\hline 80 & 2,6-Diisopropylnaphthalene & $\mathrm{C}_{16} \mathrm{H}_{20}$ & 10.300 & 0.18 \\
\hline 81 & 2-Cyclohexen-1-one, 4-(3-hydroxybutyl)-3,5,5-trimethyl- & $\mathrm{C}_{13} \mathrm{H}_{22} \mathrm{O}_{2}$ & 10.468 & 0.09 \\
\hline 82 & 6Z-2,5,5,10-Tetramethyl-undeca-2,6,9-trien-8-one & $\mathrm{C}_{15} \mathrm{H}_{24} \mathrm{O}$ & 10.502 & 0.09 \\
\hline 83 & Ar-tumerone & $\mathrm{C}_{15} \mathrm{H}_{20} \mathrm{O}$ & 10.610 & 0.50 \\
\hline 84 & 3-(4,8,12-Trimethyltridecyl) furan & $\mathrm{C}_{20} \mathrm{H}_{36} \mathrm{O}$ & 10.706 & 0.49 \\
\hline 85 & 3,3-Dimethylbutan-2-yl (E)-2-methylbut-2-enoate & $\mathrm{C}_{11} \mathrm{H}_{20} \mathrm{O}_{2}$ & 10.920 & 0.24 \\
\hline 86 & 1-Isopropenyl-3,3-dimethyl-5-(3-methyl-1-oxo-2-butenyl) Cyclopentane & $\mathrm{C}_{15} \mathrm{H}_{24} \mathrm{O}$ & 10.970 & 1.43 \\
\hline 87 & 3-Methyl-2-butenoic acid, 2-methyloct-5-yn-4-yl ester & $\mathrm{C}_{14} \mathrm{H}_{22} \mathrm{O}_{2}$ & 11.022 & 0.19 \\
\hline 88 & Fumaric acid, heptadecyl 2,2,2-trifluoroethyl ester & $\mathrm{C}_{8} \mathrm{H}_{6} \mathrm{~F}_{6} \mathrm{O}_{4}$ & 11.117 & 0.40 \\
\hline 89 & Methyl 3-(1-formyl-3,4-methylenedioxy)benzoate & $\mathrm{C}_{16} \mathrm{H}_{12} \mathrm{O}_{5}$ & 11.305 & 0.42 \\
\hline
\end{tabular}


Table 2 Chemical composition of the essential oils of T. minuta flower (Continued)

\begin{tabular}{|c|c|c|c|c|}
\hline 90 & (E)-2-Isopropyl-5-methylphenyl 2-methylbut-2-enoate & $\mathrm{Ni}$ & 11.523 & 0.52 \\
\hline 91 & 3-Methyl-2-butenoic acid, 2-methyl & $\mathrm{C}_{14} \mathrm{H}_{22} \mathrm{O}_{2}$ & 11.668 & 0.28 \\
\hline 92 & Cyclohexane, (2-nitro-2-propenyl)- & $\mathrm{C}_{9} \mathrm{H}_{15} \mathrm{NO}_{2}$ & 11.928 & 2.28 \\
\hline 93 & Hexane, 1,6-dibromo- & $\mathrm{C}_{6} \mathrm{H}_{12} \mathrm{Br}_{2}$ & 11.942 & 0.33 \\
\hline 94 & 2,2':5',2"-Terthiophene & $\mathrm{C}_{14} \mathrm{H}_{8} \mathrm{~S}_{3}$ & 12.089 & 0.25 \\
\hline 95 & Eicosane & $\mathrm{C}_{20} \mathrm{H}_{42}$ & 12.175 & 0.18 \\
\hline 96 & Benzo[b]naphtho[2,3-d]thiophene,8-dimethyl- & $\mathrm{C}_{18} \mathrm{H}_{14}$ & 12.591 & 0.90 \\
\hline 97 & 2,4-Cyclohexadien-1-one, 3,5-bis(1,1-dimethylethyl)-4-hydroxy- & $\mathrm{C}_{14} \mathrm{H}_{22} \mathrm{O}_{2}$ & 12.992 & 0.02 \\
\hline 98 & Octasiloxane, $1,1,3,3,5,5,7,7,9,9,11,11,13,13,15,15$-hexadecamethyl- & $\mathrm{C}_{16} \mathrm{H}_{48} \mathrm{O}_{7} \mathrm{Si}_{8}$ & 13.141 & 0.16 \\
\hline & Total content of EO (\%) & & & 89.16 \\
\hline & Yield of EO (\%) & & & 0.33 \\
\hline
\end{tabular}

RT Retention time, Ni not identified, EO essential oil

oil. Two fold serial dilutions were carried out under aseptic condition in sterile micro centrifuge tubes in a total volume of $100 \mu \mathrm{L}$ of Muller Hinton (MH) broth mixed with the essential oil of various concentrations ranging from $0.03-0.5 \mathrm{mg} / \mathrm{mL}$. Thereafter, $20 \mu \mathrm{L}$ of each of the inoculums dilution matching 0.5 Mc-Farland standard was added into tubes of various concentrations and votexed. Dimethyl sulfoxide (DMSO) was used as a diluting chemical solvent as reported by Okoh et al., [31]. Ciprofloxacin $(0.03-0.5 \mathrm{mg} / \mathrm{mL})$ was used as positive controls containing the organisms and solution of Ciprofloxacin while DMSO 5\% was used as negative control containing only DMSO and the various bacteria strains. The tube containing essential oil and $\mathrm{MH}$ broth was used as sterility control. The tubes were incubated at $37^{\circ} \mathrm{C}$ for $24 \mathrm{~h}$. MIC was measured by comparing the turbidity of the tubes containing bacteria and essential oil with the tube containing $\mathrm{MH}$ broth and essential oil only. MIC was expressed as the lowest concentration without bacteria growth (absence of turbidity). The viability of the bacteria strains screened was verified by inoculating $20 \mu \mathrm{L}$ of aliquots of all the tubes after incubation of various concentration on $\mathrm{MH}$ agar plates using the spread plate method, incubated at $37^{\circ} \mathrm{C}$, for $24 \mathrm{~h}$. MBC was expressed as the lowest concentration of the essential oil that prevented microbial growth on $\mathrm{MH}$ agar plates.

\section{Statistical analysis}

Antioxidant and antibacterial assays were all carried out in triplicate. The results of the essential oil were expressed

Abundance

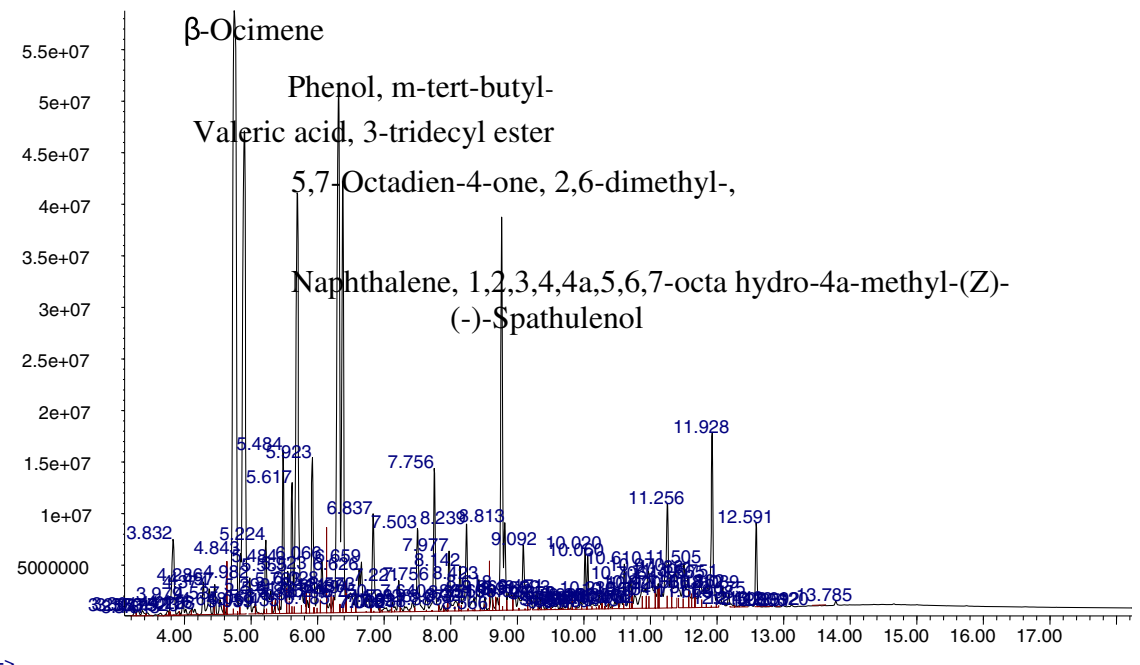

Time-->

Fig. 1 GC-MS spectra of the six major compounds present in the EO of T. minuta 
as standard deviation and correlation coefficients $\left(R^{2}\right)$ were calculated using Microsoft Excel 2007. $\mathrm{IC}_{50}$ values of the essential oil were obtained from the linear regression equation.

\section{Result}

\section{Physio-chemical characteristics of extracted essential oil} of Tegates minuta flower

The physio-chemical characteristics of the essential oil are presented in Table 1.

The yield of the essential oil was calculated in $w / \mathrm{w} \%$ (per gram) of the extracted plant sample.

\section{Chemical constituents of the essential oil of $T$. minuta extracted}

The chemical compositions of the essential oil of T. minuta flower are shown in Table 2 and the GC-MS analysis of the essential oil of T. minuta flower from this study revealed 98 compounds present in the essential oil. The major compounds were $\beta$-Ocimene $(14.40 \%), \mathrm{m}$-tert-butyl-Phenol (9.41\%), 2,6-dimethyl-, (E)-5,7-Octadien-4-one(7.14\%), 1,2, 3,4,4a,5,6,7-octa hydro-4a-methyl-naphthalene (5.58\%), and spathulenol (4.56\%) as shown in the GC-MS spectra in Fig. 1.

\section{Essential oil scavenging activity on DPPH radical}

The essential oil (EO) of T. minuta scavenging activity on the DPPH radical is as shown in Fig. 2. The EO as well as the positive control (Vitamin C) displayed concentrationdependent inhibitory effects activities on DPPH radical. The inhibitory effect of the oil at the highest concentration $(0.50 \mathrm{mg} / \mathrm{mL})$ is comparable to the inhibitory effect of the vitamin C. The essential oil of T. minuta flower displayed highest DPPH inhibitory effect of $72 \% \pm 0.012$ at $0.5 \mathrm{mg} / \mathrm{mL}$ with $\mathrm{IC}_{50}$ value of $2.45 \mathrm{mg} / \mathrm{mL}$ while vitamin $C$ displayed higher inhibitory effect $(78 \% \pm 0.002)$ on DPPH radical with $\mathrm{IC}_{50}$ value of $0.26 \mathrm{mg} / \mathrm{mL}$.

\section{Essential oil scavenging activity on ABTS radical}

The percentage ABTS inhibitory effect of the EO of $T$. minuta flower on ABTS radical are shown in Fig. 3. At $0.5 \mathrm{mg} / \mathrm{mL}$ concentration of the EO of $T$. minuta, the

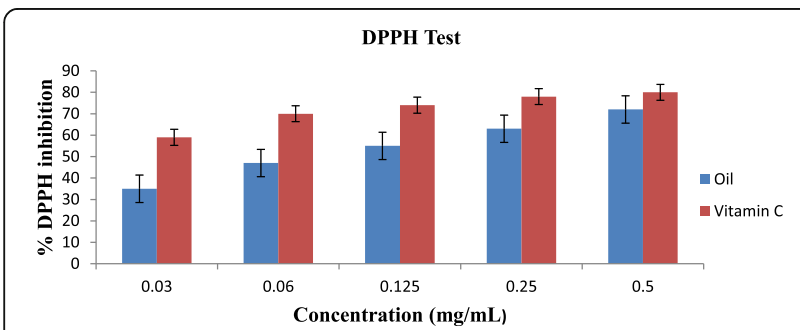

Fig. 2 Antioxidant effect of essential oil of T. minuta flower and vitamin C on DPPH radical

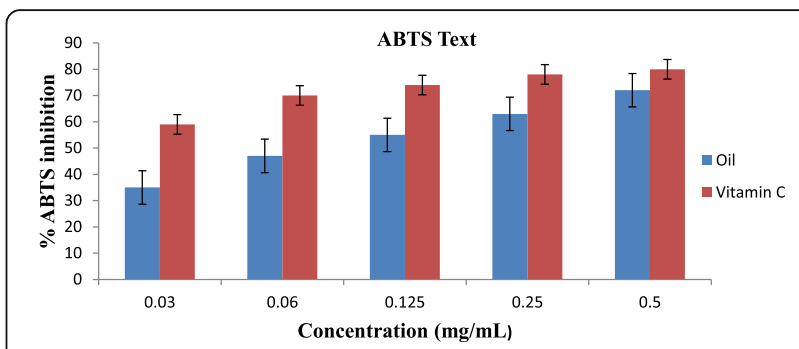

Fig. 3 Antioxidant effect of essential oil of T. minuta flower and vitamin C on ABTS radical

inhibition was $70 \%$ while vitamin C (positive control) was $80 \%$.

\section{Inhibition of lipid peroxidation}

The inhibitory action of the essential oil of $T$. minuta flower and vitamin $C$ against lipid peroxyl radical at various concentrations is shown in Fig. 4. The essential oil of $T$. minuta flower percentage inhibition of lipid peroxidation at $0.5 \mathrm{mg} / \mathrm{mL}$ was $67 \%$ while vitamin $C$ was $54 \%$ indicating lower inhibitory scavenging effect compared to the results obtained in DPPH and ABTS tests at same concentration.

\section{MIC of the essential oil of T. muinuta flower}

The essential oil of T. minuta flower demonstrated good antibacterial activity against all the test bacteria strains. The minimum inhibitory concentration (MIC) value of $0.06 \mathrm{mg} / \mathrm{mL}$ was exhibited for Vibrio spp., E. coli, E. cloacae and $L$. ivanovii, while the MIC $(0.125 \mathrm{mg} / \mathrm{mL})$ for the EO against $S$. aurius, $M$. smegatis and $S$. uberis was higher as shown in Table 3.

\section{MBC of the essential oil}

The essential oil of $T$. minuta flower displayed incredible antibacterial activities against Gram-negative bacteria test strains (E. cloacae, Vibrio spp. and E. coli) and Gram-positive bacteria (S. uberis) at varied concentrations as showed in Table 4 . The minimum bactericidal

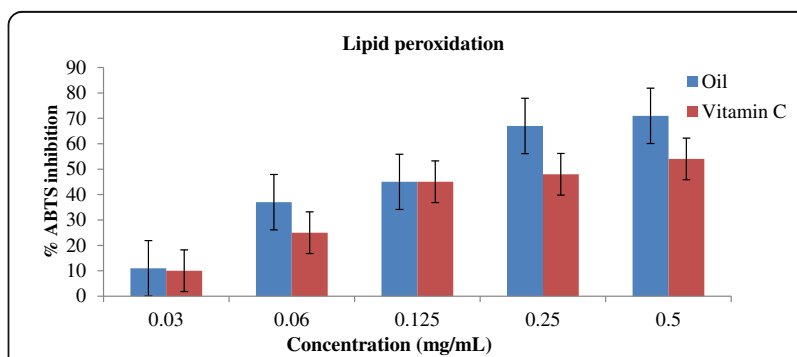

Fig. 4 Antioxidant effect of essential oil of T. minuta flower and vitamin $C$ on lipid peroxidation radical 
Table 3 Minimum inhibitory concentrations (MIC) of essential oil of T. minuta flower

\begin{tabular}{|c|c|c|c|c|c|c|c|c|c|c|c|c|}
\hline \multirow{3}{*}{$\begin{array}{l}\text { Bacteria } \\
\text { Gram positive }\end{array}$} & \multicolumn{12}{|c|}{ Concentrations of essential oil (mg/mL) and MIC (mg/mL) } \\
\hline & \multicolumn{2}{|c|}{0.5} & \multicolumn{2}{|c|}{0.25} & \multicolumn{2}{|c|}{0.125} & \multicolumn{2}{|c|}{0.06} & \multicolumn{2}{|c|}{0.03} & \multicolumn{2}{|c|}{$\mathrm{MIC}(\mathrm{mg} / \mathrm{mL})$} \\
\hline & A & B & A & B & A & B & A & B & A & B & Oil & Ciprofloxacin \\
\hline S. aureus & - & - & - & - & - & - & + & - & + & - & 0.125 & 0.00 \\
\hline L. ivanovii & - & - & - & - & - & - & - & - & + & - & 0.06 & 0.00 \\
\hline M. smegatis & - & - & - & - & - & - & + & - & + & + & 0.125 & 0.06 \\
\hline S. uberis & - & - & - & - & - & - & + & - & + & - & 0.125 & 0.00 \\
\hline \multicolumn{13}{|l|}{ Gram negative } \\
\hline E. cloacae & - & - & - & - & - & - & - & - & + & - & 0.06 & 0.00 \\
\hline E. coli & - & - & - & - & - & - & - & + & + & + & 0.06 & 0.125 \\
\hline Vibro spp. & - & - & - & - & - & + & - & + & + & + & 0.06 & 0.25 \\
\hline
\end{tabular}

Key: $\mathrm{A}=$ Essential oil, $\mathrm{B}=$ ciprofloxacin, $+=$ Growth, $-=$ no growth

concentration of the $\mathrm{EO}$ and positive control are shown in Table 4 below.

\section{Discussion}

The colour and fragrance of the extracted essential oil of T. minuta flower obtained in this study were similar to previous report of Wanzala \& Ogoma, [32]. Previous study done by Chamorro et al., [33] on the EO from Tagetes minuta flower showed that $\beta$-Ocimene was reported having the highest chemical content of the EO of T. minuta flower and this agrees with our result. However, study done by Shirazi et al., [34] and Garcia et al. [35], reported the predominance of dihydrotagetone in the essential oil of $T$. minuta which was not found in the GC-MS result of the EO of T. minuta in our study. Several studies have shown that the chemical composition of the extracted essential oil of $T$. minuta varied according to the location where it was harvested, the growth stage at which it was harvested and the part of the plant used for the extraction [36]. The chemical composition from the GC-MS analysis of the EO of T. minuta plant grown in Kenya do not reveal some of the compounds present in our result as reported by Kyarimpa et al. [37], as well as those grown in Argentina [36].

Differences in the chemical constituents of Tagetes oil has also been allotted to some environmental variables like soil, temperature and the total period of exposure to sunlight [38]. The differences in the chemical constituents of the EO of T. minuta flower could be attributed to many factors which could include but not limited to location, stage of cultivation, season of cultivation and part of the plant used. The chemical composition of the essential oil of $T$. mimuta flower shows various classes of terpenes ranging from hemiterpenes to sesquiterpenes. Similar study done by Wanzala \& Ogoma, [32] showed that $T$. minuta essential oil contains a wide range of secondary metabolites mainly sesquiterpenes and monoterpenes including $\beta$-ocimene, camphene and bicyclogermacrene and their report corroborates with our result. At $0.5 \mathrm{mg} / \mathrm{mL}$ concentration, the $\mathrm{EO}$ was $72 \%$ while vitamin $\mathrm{C}$ was $76 \%$ and the $\mathrm{EO}$ of $T$. minuta flower displayed lower DPPH radical scavenging activity at various concentrations compared to the vitamin $\mathrm{C}$ as

Table 4 Minimum bactericidal concentrations (MBC) of essential oil of T. minuta flower

\begin{tabular}{|c|c|c|c|c|c|c|c|c|c|c|c|c|}
\hline \multirow{3}{*}{$\begin{array}{l}\text { Bacteria } \\
\text { Gram positive }\end{array}$} & \multicolumn{12}{|c|}{ Concentrations of essential oil $(\mathrm{mg} / \mathrm{mL})$ and $\mathrm{MBC}(\mathrm{mg} / \mathrm{mL})$} \\
\hline & \multicolumn{2}{|c|}{0.5} & \multicolumn{2}{|c|}{0.25} & \multicolumn{2}{|c|}{0.125} & \multicolumn{2}{|c|}{0.06} & \multicolumn{2}{|c|}{0.03} & \multicolumn{2}{|c|}{$\mathrm{MBC}(\mathrm{mg} / \mathrm{mL})$} \\
\hline & A & B & A & B & A & B & A & B & A & B & Oil & Ciprofloxacin \\
\hline S. aureus & + & - & + & - & + & - & + & - & + & + & 0.00 & 0.06 \\
\hline L. ivanovii & + & - & + & - & + & - & + & - & + & - & 0.00 & 0.00 \\
\hline M. smegatis & + & - & + & - & + & - & + & - & + & + & 0.00 & 0.06 \\
\hline S. uberis & - & - & + & - & + & - & + & - & + & - & 0.5 & 0.00 \\
\hline \multicolumn{13}{|l|}{ Gram negative } \\
\hline E. cloacae & - & - & - & - & - & - & - & - & + & - & 0.06 & 0.00 \\
\hline E. coli & - & - & - & - & - & - & - & + & + & + & 0.06 & 0.125 \\
\hline Vibro spp. & - & - & - & - & - & + & + & + & + & + & 0.125 & 0.25 \\
\hline
\end{tabular}

Key: A = oil, B = ciprofloxacin, + = Growth, - = no growth 
shown in Fig. 2. Our result is similar with the report of Muyima et al., [3] on inhibitory effect of the essential oil of T. minuta against DPPH radical. The action implicated in antioxidant activity assay is the capacity of the molecule to release hydrogen atom to a radical which is the key factor that is involved in free radical scavenging activity $[27,39]$. This effect is displayed as the colour DPPH• fades away (purple to yellow) in the test solution due to the production of neutral DPPH-H molecule upon absorption of hydrogen atom from an antiradical [40]. The sample antioxidant strength is established by the decreased of UV absorption at $517 \mathrm{~nm}$. Study has shown that DPPH method is not an exact radical specie assay but a general radical scavenging strength of an antioxidant compound [41].

For the assumed antioxidant strength of the essential oil of T. minuta flower, we used a mono-cation (ABTS radical) and one specific type of specie, the lipid peroxyl radical. The antioxidant activities of the EO of T. minuta flower and vitamin $\mathrm{C}$ were concentrations dependent as observed in DPPH Test. However, a lower radical scavenging effect of $70 \%$ was displayed by $\mathrm{EO}$ on ABTS radical at highest concentration $(0.50 \mathrm{mg} / \mathrm{mL})$ while vitamin $\mathrm{C}$ had higher effect of $80 \%$ compared to the DPPH experiment as shown in Fig. 3. The $\mathrm{IC}_{50}$ values of $2.76 \mathrm{mg} / \mathrm{mL}$ and $1.14 \mathrm{mg} / \mathrm{mL}$ for $\mathrm{EO}$ flower of $T$. minuta and vitamin $\mathrm{C}$ were obtained from the linear regression equation from the graph. Percentage lipid peroxidation inhibitory activity of the EO was higher than that of vitamin $C$ (standard drug) and the inhibitory effects was dose dependent. The essential oil of T. minuta flower exhibited the highest lipid peroxidation inhibitory effect of $71 \% \pm 0.001$ at the concentration of $0.5 \mathrm{mg} / \mathrm{mL}$ with $\mathrm{IC}_{50}$ value of $3.23 \mathrm{mg} / \mathrm{mL}$ while vitamin $\mathrm{C}$ displayed lower percentage lipid peroxidation inhibitory effects of $54 \% \pm 0.004$ with $\mathrm{IC}_{50}$ value of $4.19 \mathrm{mg} / \mathrm{mL}$ as shown in Fig. 4. The result implies that the EO of T. minuta flower possess higher lipid peroxidation properties than vitamin C. Antiradical scavenging action of the essential oils may be credited to the substitution of hydroxyl groups of the aromatic ring systems of the phenolic compounds due to their hydrogen giving capacity [42]. Our results in this study demonstrate the ability of the EO of T. minuta flower to scavenge three different radicals suggesting it usefulness as a good antioxidant agent for further investigation.

The MIC values of the EO of T. minuta against the test bacteria strains as shown in Table 3 displayed that the EO was more active against Gram-negative than Gram-positive bacteria and this report is not in agreement with the report of Senatore et al., [43] and they reported that the MIC value of the EO of T. minuta from UK for Gram-positive bacteria were $6.25-25 \mu \mathrm{g} / \mathrm{mL}$ and 25-50 $\mu \mathrm{g} / \mathrm{mL}$ for Gram-negative bacteria. Minimum bactericidal concentration of any test sample is the lowest concentration of antimicrobial agents capable of killing or preventing any visible bacteria growth after twenty four hours of incubation under standardized sets of conditions [44, 45]. The EO showed MBC value of $0.06 \mathrm{mg} / \mathrm{mL}$ for $E$. coli and E. cloacae while at higher concentration of $0.125 \mathrm{mg} / \mathrm{mL}$, it was bactericidal against Vibrio spp. and the $\mathrm{MBC}$ value for S. uberis was recorded at $0.5 \mathrm{mg} / \mathrm{mL}$ (Table 4). The essential oil of $T$. minuta flower had greater bactericidal effect against $S$. uberis, Vibro spp. and E. coli. This suggests that the essential oil of $T$. minuta flower may contain some bioactive compounds that could be efficacious in the prevention and treatment of infectious diseases that are linked to these organisms resistant to some antibiotics.

\section{Conclusion}

The results in this present study shows that apart from traditional applications of $T$. minuta plant, the essential oil contained vast bioactive constituents and could serve as a potent resource for new antibacterial and antioxidant agent. However, further studies are required to isolate the main active components, evaluate the bioactivities in-vivo and toxicity of the essential oil of T. minuta flower.

\section{Acknowledgements \\ We would like to acknowledge South Africa National Research Foundation (NRF) for Funding this research and the University of Fort Hare for providing} conducive environment.

\section{Funding}

The author's are grateful to National Research Foundation for financing the project.

Availability of data and materials

All data were provided in the paper.

\section{Authors' contributions}

Al conducted the research and wrote the manuscript; $\mathrm{BCl}$ designed the research, sought for funding and supervised the work; UUN edited the manuscript; SOO proofread the manuscript; AIO and LCO corrected the final manuscript. All authors read and approved the final manuscript.

Ethics approval and consent to participate

Ethical approval was granted by Govan Mbeki Research \& Development Centre (GMRDC),

University of Fort Hare (UFH) and consent was approved by Cala Traditional Health Practitioners.

\section{Consent for publication}

Not applicable.

\section{Competing interest}

The authors declare they have no competing interest.

\section{Publisher's Note}

Springer Nature remains neutral with regard to jurisdictional claims in published maps and institutional affiliations.

\section{Author details}

'SAMRC Microbial Water Quality Monitoring Centre, University of Fort Hare, Alice 5700, South Africa. ${ }^{2}$ Applied and Environmental Microbiology Research Group (AEMREG), Department of Biochemistry and Microbiology, University of Fort Hare, Private Bag X1314, Alice, Eastern Cape Province 5700, South

Africa. ${ }^{3}$ Academic and Research Division, University of Fort Hare, Private Bag X1314, Alice, Eastern Cape Province 5700, South Africa. 
Received: 8 May 2017 Accepted: 26 June 2017

Published online: 05 July 2017

\section{References}

1. Zia-Ul-Haq M, Riaz M, Saad B. Anthocyanins and human health: Biomolecular and therapeutic aspects. Spring Intern Publi. 2016;

2. Sies H. Antioxidants in disease, mechanisms and therapy. New York Academ: Press; 1996

3. Muyima NYS, Nziweni S, Mabinya LV. Antimicrobial and antioxidative activities of Tagetes minuta, Lippia javanica and Foeniculum vulgare essential oils from the Eastern Cape Province of South Africa. J Essen Oil Bear Plants. 2004; $7(1): 68-78$

4. Chatterjee S, Poduval TB, Tilak JC, Devasagayam TPA. A modified, economic, sensitive method for measuring total antioxidant capacities of human plasma and natural compounds using Indian saffron (Crocus sativus). Clin Chim Acta. 2005;352:155-63.

5. Gupta D, Dubey J, Kumar M. Phytochemical analysis and antimicrobial activity of some medicinal plants against selected common human pathogenic microorganisms. Asian Pac J Trop Dis. 2016;6(1):15-20.

6. Ndamane Y, Kambizi L, Songca SP, Oluwafemi OS. Antibacterial effectiveness of Tetradenia riparia extract, a plant traditionally used in the Eastern Cape Province to treat diseases of the respiratory system. J Med Plants Res. 2013;7(37):2755-60.

7. Guschin A, Ryzhikh P, Rumyantseva T, Gomberg M, Unemoe M. Treatment efficacy, treatment failures and selection of macrolide resistance in patients with high load of Mycoplasma genitalium during treatment of male urethritis with Josamycin. BMC Infect Dis. 2015;15(40):1-7.

8. Balouiri M, Sadiki M, Ibnsouda SK. Methods for in vitro evaluating antimicrobial activity: a review. J Pharm Anal. 2016:1-9.

9. Priecina L, Karklina D. Natural antioxidant changes in fresh and dried spices and vegetables. Intern J Innova Res Sci Eng. 2014;8:4804.

10. Oshaghi EA, Khodadadi I, Tavilani H, Goodarzi MT. Aqueous extract of Anethum graveolens $\mathrm{L}$. has potential antioxidant and antiglycation effects. Iran J Med Sci. 2016;41(4):328-33.

11. Cos P, Vlietinck AJ, Berghe DV, Maes L. Anti-infective potential of natural products: how to develop a stronger in vitro 'proof-of-concept'. J Ethno. 2006;106:290-302.

12. Sasidharan S, Chen Y, Saravanan D, Sundram KM, Latha LY. Extraction, isolation and characterization of bioactive compounds from plants' extracts. Afric J Trad Compl Altern Med. 2011;8(1):1-10.

13. Ahmad I, Beg AZ. Antimicrobial and phytochemical studies on 45 Indian medicinal plants against multi-drug resistant human pathogens. J Ethnopharmacol. 2001;74:113-23.

14. Nweze El, Okafor Jl. Activities of a wide range of medicinal plants and essential oil vs. Scedospaorium isolates. Am Eurasian J Res. 2010:5:161-9.

15. Ravi KU, Shoeb A. Ethno-medicinal plants and their pharmaceutical potential. J Pharm Res. 2012;5(4):2162-73.

16. Calo JR, Crandall PG, O'Bryan CO, Ricke SC. Essential oils as antimicrobials in food systems - a review. Food Cont. 2015;54:111-9.

17. Andrade BFMT, Barbosa LN, Probst I. Da S. \& Júnior a.F. Antimicrobial activity of essential oils. J Essent Oil Res. 2013;26(1):34-40.

18. Chaves AV, He ML, Yang WZ, Hristov AN, McAllister TA, Benchaar C. Effects of essential oils on proteolytic, deaminative and methanogenic activities of mixed ruminal bacteria. Canad J Anim Sci. 2008;88:117-22.

19. Dasgupta N, Ranjan S, Saha P, Jain R, Malhotra S, Saleh MAAM. Antibacterial activity of leaf extract of Mexican marigold (Tagetes erecta) against different gram positive and gram negative bacterial strains. J Pharm Res. 2012;5(8): 4201-3.

20. Soule JA. Medicinal and beverage uses of Tagetes (Tageteae: Compositae). Amer J Bot. 1993;80:177.

21. Negahban M, Msaada K, Tafazoli E, Zakerin A. Effect of foliar application of diammonium phosphate on morphological characteristics and constituents of essential oil of Mexican marigold (Tagetes minuta L.). Med Arom Plant Sci Biotech. 2013;7:11-8.

22. Shahzadi I, Hassan A, Khan UW, Shah MM. Evaluating biological activities of the seed extracts from Tagetes minuta L. found in northern Pakistan. J Med Plants Res. 2010;4:2108-12.

23. Nqeno N, Chimonyo M, Mapiye C, Marufu MC. Ovarian activity, conception and pregnancy patterns of cows in the semiarid communal rangelands in the Eastern Cape Province of South Africa. Anim Reprod Sci. 2010;118:140-7.
24. Omoruyi BO, Afolayan AJ, Bradley G. The inhibitory effect of Mesembryanthemum edule (L.) bolus essential oil on some pathogenic fungal isolates. BMC Complem Altern Med. 2014;14(168):1-7.

25. Ajileye OO, Obuotor EM, Akinkunmi EO, Aderogba MA. Isolation and characterization of antioxidant and antimicrobial compounds from Anacardium occidentale L. (Anacardiaceae) leaf extract. J King Saud Univ Sci. 2015;27:244-52.

26. Kannan RRR, Arumugam R, Thangaradjou T, Anantharaman P. Phytochemical constituents, antioxidant properties and p-coumaric acid analysis in some sea grasses. Food Res Int. 2013;54:1229-36.

27. Badmus AJ, Adedosu TO, Fatoki JO, Adegbite VA, Adaramoye OA, Odunola OA. Lipid peroxidation inhibition and antiradical activities of some leaf fractions of Mangifera indica. Acta Polon Pharm Drug Res. 2011;68(1):23-9.

28. Clinical and Laboratory Standards Institute. Performance standards for antimicrobial susceptibility testing; twenty-fourth informational supplement; 2014.

29. Duarte MC, Figueira GM, Sartoratto A, Rehder VL, Delarmelina C. AntiCandida activity of Brazilian medicinal plants. J Ethnopharmacol. 2005;97(2): 305-11.

30. Gullon B, Pintado ME, Perez-Alvarez JA, Viuda-Martos M. Assessment of polyphenolic profile and antibacterial activity of pomegranate peel (Punica granatum) flour obtained from co-product of juice extraction. Food Cont. 2016;59:94-8.

31. Okoh OS, Iweriebor BC, Okoh OO, Nwodo UU, Okoh Al. Antibacterial and antioxidant properties of the leaves and stem essential oils of Jatropha gossypifolia L. BioMed Res Intern. 2016:1-9.

32. Wanzala W, Ogoma SB. Chemical composition and mosquito repellency of essential oil of Tagetes minuta from the southern slopes of mount Elgon in western Kenya. J Essent Oil Bear Plants. 2013;16(2):216-32.

33. Chamorro ER, Ballerini G, Sequeira AF, Velasco GA, Zalazar MF. Chemical composition of essential oil from Tagetes minuta $L$. leaves and flowers. J Argent Chem Soc. 2008;96(1-2):80-6.

34. Shirazi MT, Gholami H, Kavoosi G, Rowshan V, Tafsiry A. Chemical composition, antioxidant, antimicrobial and cytotoxic activities of Tagetes minuta and Ocimum basilicum essential oils. Food Sci Nutr. 2014;2(2):146-55.

35. Garcia MV, Matias J, Barros JC, de Lima DP, Lopes RDS, Andreotti R. Chemical identification of Tagetes minuta Linnaeus (Asteraceae) essential oil and its acaricidal effect on ticks. Rev Bras Parasit Vet. 2012;21(4):405-11.

36. Gil A, Ghersa CM, Leicach S. Essential oil yield and composition of Tagetes minuta accessions from Argentina. Biochem Syst Ecol. 2000;28:261-74.

37. Kyarimpa MC, Böhmdorfer S, Wasswa J, Kiremire BT, Ndiege IO, Kabasa JD. Essential oil and composition of Tagetes minuta from Uganda. Larvicidal activity on Anopheles gambiae. Indust Crops Prod. 2014;62:400-4.

38. Yankeu SY, Vermaak I, Viljoen AM, Sandasi M, Kamatou GPP. Essential oil variation of Tagetes minuta in South Africa -a chemometric approach. Biochem Syst Ecol. 2013;51:320-7.

39. Miliauskas $G$, Venskutonis PR, van Beek TA. Screening of radical scavenging activity of some medicinal and aromatic plant extracts. Food Chem. 2004;85: 231-6.

40. Valko M, Leibfritz D, Moncol J, Cronin MTD, Mazur M, Telser J. Free radicals and antioxidants in normal physiological functions and human disease. Intern J Biochem Cell Biol. 2007;39(1):44-84.

41. Guerrini A, Sacchetti G, Rossi D, Paganetto G, Muzzoli M, Andreotti E, Tognolini M, Maldonado ME, Bruni R. Bioactivities of Piper aduncum L. and Piper obliquum Ruiz \& Pavon (Piperaceae) essential oils from Eastern Ecuador. Environ Toxicol Pharm. 2009;27(1):39-48.

42. Mahmoud Gl. Biological effects, antioxidant and anticancer activities of marigold and basil essential oils. J Med Plants Res. 2013;7(10):561-72.

43. Senatore F, Napolitano F, A-H MM, Harris PJC, Mnkeni PNS, Henderson J. Antibacterial activity of Tagetes minuta L. (Asteraceae) essential oil with different chemical composition. Flavour Fragr J. 2004;19:574-8.

44. Shahidi BGH. Evaluation of antibacterial properties of Iranian medicinal plants against Micrococcus aureus, Serratia marcescens, Klebsiella pneunomiae and Bordella bronchoseptica. Asian J Sci. 2004;3(1):82-6.

45. Kaur S, Mondal P. Study of total phenolic and flavonoid content, antioxidant activity and antimicrobial properties of medicinal plants. J Microbiol Experim. 2014;1(1):4-6 